réactifs de Storch, un tel lait a toujours une teinte un peu plus rougeâtre que le lait normal. Nous a vons aussi remarqué que quand du lait avait été chauffé tellement rapidement qu'il y avait possibilité qu'il donne une réaction de Storch positive après repos, ce n'était pas le lait chauffé le plus bas qui donnait la teinte la plus forte. Il est probable que les leucoeytes abandonnent plus facilement la peroxydase non encore détruite, quand ils sont eux-mêmes très touchés par le chauffage.

Comme ce n'est que le chauffage très court qui peut causer des difficultés lors du contrôle de la pasteurisation, le mieux serait de munir tous les systèmes régénérateurs d'un chambreur, et pour ceci, milite également, le fait suivant : si le chauffage est si court qu'il ne peut pas détruire la peroxydase incluse dans les leucocytes, on peut craindre que les bactéries de la tubereulose qui sont incluses dans la boue et les débris de tissu, ne soient également pas détruites, et c'est pourtant là le but de la pasteurisation. C'est pourquoi on peut considérer qu'il est raisonnable d'exiger que le lait pasteurisé par pasteurisation haute donne aussi après un certain repos une réaction de Storch négative. En ce qui concerne le lait destiné à la consommation, on ne peut, en aucun cas, échapper à cette exigence.

\title{
L'AMÉLIORATION DE LA FABRICATION DE LA CASÉINE
}

\author{
par G. GENIN
}

Ingénieur chimiste E. P. C. I.

Le lait écrémé est le sous-produit le plus important de l'industrie laitière et la caséine est la substance la plus intéressante que l'on extrait de ce lait écrémé. L'extraction de la easéine du lait semble à première vue très simple, mais o'est justement ce manque de complication apparente qui a trompé beaucoup d'expérimentateurs qui ont voulu voir dans la précipitation de la caséine un phénomène extrêmement élémentaire. La précipitation de la caséine dans le lait écrémé s'effectue industriellement soit par addition d'acide, soit par fermentation naturelle donnant naissance aux acides nécessaires pour la coagulation de la caséine (nous mettons volontairement de côté la précipitation par la présure).

Il est si facile de laisser surir le lait ou de lui ajouter la quantité d'acide juste nécessaire pour entraîner la précipitation de la caséine, qu'on oublie très fréquemment la complexité chimique et physique de la substance que l'on cherche à obtenir et les variations possibles que peut subir la qualité de ee produit suivant les conditions de sa préparation. Ce sont pourtant ces variations dans les caractéristiques 
du produit qui ont entraîné de très grandes difficultés, en particulier dans l'industrie du papier qui est une des plus importantes consommatrices de caséine. On sait en effet que la caséine sert à glacer le papier que l'impression moderne utilise aujourd'hui. Les mêmes difficultés se rencontrent d'ailleurs dans les applications de la caséine à la fabrication des colles, des peintures à l'eau et détrempes, des produits servant à glacer le cuir, des produits d'entretien, des matières plastiques à base de caséine, des produits alimentaires et produits pharmaceutiques spéciaux, des toiles cirées, du linoléum, des savons, etc... qui toutes utilisent la caséine. Il est étonnant à première vue qu'une réaction aussi simple qu'une précipitation par l'acide puisse donner naissance à des produits essentiellement variables, donnant au cours de leur emploi des résultats très différents.

La raison en est due en partie à ce que la caséine, comme d'ailleurs beaucoup d'autres produits, est un corps d'une complexité beaucoup plus grande que la simplieité de son nom semble l'indiquer. La caséine est en réalité constituée par un grand nombre de corps protéiniques mélangés intimement. Or, la première qualité que l'on exige d'un produit employé dans l'industrie et en particulier dans celles que nous venons de nommer, est sa constance. Par conséquent, il est indispensable que l'acidification du lait, obtenue soit par fermentation, soit par addition d'acide, puisse être conduite dans des conditions d'une exactitude absolue, afin de parvenir à un produit final toujours constant. Pour obtenir ce résultat, le point capital est de contrôler l'acidité qui produira la précipitation. Cette acidité se détermine aujourd'hui le plus exactement par la mesure du $p \mathrm{H}$. Il faut également que l'opération soit conduite de telle sorte que le pourcentage de cendres dans la caséine soit réduit au minimum.

Récemment, un brevet américain dû à M. SHEFFIELd a été publié [1]; il décrit un procédé continu de préparation de la caséine qui fait intervenir certains faits nouveaux permettant de contrôler les facteurs qui agissent sur la précipitation et sur le traitement ultérieur de la caséine. Il permet done d'obtenir une substance supérieure par son uniformité, ce qui justifie le prix plus élevé de la caséine ainsi produite. M. SHEFFIELD a pris d'autres brevets actuellement en discussion, mais le principe de son procédé a été étudié récemment dans une publication américaine à laquelle nous emprunterons le principal de cette note [2].

Pour bien mettre en relief ce qui fait la nouveauté du procédé Sheffield, il importe de rappeler en quelques mots les caractéristiques des procédés anciens et de résumer les caractéristiques physicochimiques de la caséine. Nous renverrons nos lecteurs qui seraient 
intéressés par cette question et qui seraient désireux d'avoir des renseignements plus complets à la remarquable étude que $\mathrm{M}$. BEAU a fait paraître sur la question [3].

Le lait est normalement légèrement acide, puisque son $p H$ est d'environ 6,6. Il est constitué par une suspension colloïdale de protéine et d'albumine dans une solution aqueuse de lactose et de certains sels minéraux. La méthode la plus simple pour récupérer la caséine consiste done à obtenir, au moyen de microorganismes naturellement présents, de l'acide lactique aux dépens du lactose et de précipiter ainsi la caséine. A cet effet, le lait écrémé est àbandonné dans des euves et maintenu à une température de $37^{\circ} \mathrm{C}$. environ qui favorise la ferme.ttation. Lorsque l'acidité a atteint une valeur correspondant à un $p H$ de 4,6, la quantité d'acide lactique produite est suffisante pour obtenir une caillebotte de la structure désirable. Celle-ci, après avoir été désagrégée soit au moyen de palettes, soit par chauffage, est séparée du sérum par décantation. On lave la caillebotte à l'eau deux ou trois fois et on la presse dans des presses hydrauliques ou dans des presses à main, afin d'enlever la plus grande partie de l'humidité. Les gâteaux sont ensuite broyés, étendus sur des elaies et séchés dans une étuve. Finalement, on pulvérise finement la caséine après séchage.

C'est cette méthode de fabrication, comme on le voit très simple, qui est encore utilisée en Argentine et qui, aussi étrange que cela soit, donne une qualité supérieure de caséine. Ses principaux inconvénients résultent de la fermentation elle-même et de la présence dans la caillebotte de différentes impurètés qu'il est difficile d'éliminer par un procédé économique.

L'autre procédé de préparation de la caséine correspond plus aux méthodes modernes et consiste à ajouter un acide minéral dilué, le plus souvent l'acide sulfurique et parfois également l'acide chlorhydrique, au lait écrémé, à mélanger le tout soigneusement de façon à précipiter la caillebotte, puis à la traiter comme dans le eas précédent. Ce procédé présente les mêmes inconvénients que le procédé par fermentation naturelle, à l'exception du temps perdu. Par contre, il présente un autre inconvénient, e'est l'absence d'uniformité du produit. En effet, au cours de l'addition de l'acide et malgré tout le soin apporté à eette opération, on ne peut pas éviter qu'à un certain moment, une certaine partie du lait écrémé soit en présence d'une plus grande quantité d'acide que d'autres et la caséine précipitée contient de ce fait une plus ou moins forte proportion de protéines étrangères.

Le manque d'uniformité du prođuit ainsi obtenu l'a donc fait considérer comme étant de qualité inférieure et, pour cette raison, son prix sur le marché de la caséine est sensiblement inférieur. 
En résumé, quel que soit le procédé employé, toutes les méthodes qui travaillent en discontinu présentent des difficultés sérieuses. Des progrès certes ont été faits au cours de ces dernières années, mais insuffisants encore au point de vue de l'uniformité du produit final.

\section{Propriétés physico-chimiques de la caséine.}

La caséine, tout en étant un produit très pur, appartient à un des groupes les plus complexes des protéines : les phosphoprotéines. Elle contient du carbone, de l'oxygène, de l'azote, de l'hydrogène, du phosphore et du soufre et, comme nous l'avons dit, existe dans le lait sous la forme d'une suspension colloïdale combinée avec de faibles proportions de calcium. Ce produit vort sa complexité encore augmentée par la présence des autres constituants du lait. Si on coagule donc un tel mélange, il est normal de penser que les variations de l'acidité vont entraîner des variations dans la composition du mélange précipité. En effet, l'addition d'une très faible proportion d'acide ne peut être faite sans qu'au même instant on dépasse l'acidité optimum pour certains constituants ou au contraire qu'on soit en deçà de l'acidité optimum pour d'autres constituants. L'acidité ajoutée n'ayant pas seulement le rôle de neutralisant à jouer mais étant en même temps absorbée ou adsorbée par le présipité, il en résulte qu'un mélange incomplet conduit à un produit hétérogène.

La caillebotte précipitée qui renferme une plus ou moins grande proportion d'acide est, comme nous l'avons vu, lavée et séchée afin d'éliminer la majeure partie de l'humidité. Cette opération n'est évidemment pas parfaite et elle laisse subsister dans la caséine des traces irrégulièrement distribuées d'acide. Au cours du séchage, l'acide résiduaire hydrolyse la caséine dans des proportions plus ou moins grandes suivant la concentration de l'acide dans les différents points du produit.

On voit done, alors que l'industrie du papier exige par exemple une caséine dont les propriétés soient rigoureusement uniformes, qu'il est, par les procédés que nous venons de décrire, presque impossible d'obtenir un produit d'une uniformité suffisante. C'est dans le but de surmonter ces difficultés que l'ingénieur américain dont nous avons cité le nom au début de cette étude est parvenu à mettre au point un procédé continu de fabrication de la caséine qui permet, grâce à sa continuité, d'obtenir un produit beaucoup plus constant.

\section{Nouveau procédé de préparation de la caséine.}

Tout ce que nous venons de dire peut se résumer en indiquant que le contrôle de l'acidité est le facteur principal de la fabrication 
de la caséine, autrement dit le récipient dans lequel se fait le mélange de l'acide et du lait constitue la partie vitale de l'installation. Dans le nouveau procédé américain, ce mélange s'effectue dans un récipient en grès muni de chicanes dans lesquelles s'écoulent à des vitesses rigoureusement contrôlées le lait et l'acide en solution. De cette façon, l'acidification de chaque portion de lait s'effectue en une fraction de seconde et la répartition des chicanes permet de réaliser un mélange absolument uniforme des deux courants liquides. Aucune portion du lait ne se trouve au contact d'une quantité d'acide supérieure à celle qui est nécessaire pour amener le $p H$ à la valeur désirée. Le mélange s'effectue en utilisant d'une part de l'acide chlorhydrique de densité 1,20 dilué avec 4 fois son volume d'eau et du lait écrémé préalablement réchauffé à $44^{\circ}$.

La précipitation complète de la caséine exige un temps plus important que le mélange proprement dit de l'acide et du lait, aussi ee mélange, après avoir quitté la chambre de mélangeage, circulera-t-il dans une série de récipients munis également de chicanes qui favorisent l'agitation du produit jusqu'à ce que la eaillebotte se soit parfaitement séparée du sérum. Cette opération, comme le mélange proprement dit, s'effectue dans des récipients en grès.

Le sérum qui contient encore certains constituants de valeur est séparé de la caillebotte sur un plan incliné muni d'une vis transporteuse. La caillebotte qui se sépare est immédiatement évacuée par la vis et le sérum clair s'écoule d'une façon continue par un trop-plein disposé dans le récipient.

Le sérum contient encore du lactose, des sels inorganiques et une petite proportion d'albumine. On peut l'évaporer directement de façon à obtenir une poudre pouvant servir à la nourriture de la volaille; on peut en extraire le lactose, on peut par fermentation l'utiliser comme source d'acide lactique, on peut enfin séparer ses constituants albumineux.

Au cours du dépôt proprement dit, la caillebotte encore chaude présente une certaine tendance à s'agglomérer. Après égouttage, les gros grumeaux sont placés dans un désintégrateur qui est constitué essentiellement par un eylindre métallique perforé dans lequel tourne un axe portant un certain nombre de bras qui brisent les grumeaux en petits morceaux, en même temps qu'ils sont soumis à l'action d'un courant d'eau froide.

La caillebotte désintégrée est à nouveau lavée sur un plan incliné en métal perforé au moyen d'un courant d'eau froide. Là encore une vis transporteuse élève la caillebotte en même temps qu'elle est lavée, de telle sorte qu'à la partie supérieure du laveur, on obtient une easéine pratiquement exempte de matières solubles dans l'eau. 
Ce procédé de lavage continu et en même temps très lent, donne une caséine en petits grumeaux ne contenant qu'un très faible excès d'acidité et de cendres.

La caséine sous cette forme est alors dirigée dans une presse continue de conception tout à fait nouvelle qui expulse la majeure partie de l'eau et laisse subsister un produit renfermant $50 \%$ d'humidité. Cette presse est constituée essentiellement par deux rouleaux entre lesquels passe la caséine que l'on veut assécher. L'un de ces rouleaux est massif et chromé, l'autre est en bronze perforé. Au cours du passage entre les deux rouleaux, l'eau expulsée pénètre dans le cylindre perforé et est évacuée. Le gâteau continu de caséine adhère à ce cylindre perforé, mais il en est décollé au moyen d'un racloir en bois.

Finalement, on obtient donc une caséine floculante relativement poreuse et qui est prête à être séchée. On peut évidemment utiliser à cet effet l'outillage habituel, toutefois le séchage sur plateaux présente l'inconvénient de former à la surface des gâteaux de caséine une peau qui s'oppose à l'évacuation de l'eau interne et qui favorise le développement de l'hydrolyse. On a constaté qu'on obtient des résultats bien supérieurs en projetant les particules finement divisées de caséine dans un courant d'air chaud et sec. Ces particules étant relativement petites, leur séchage est très rapide et les phénomènes d'hydrolyse sontréduits au strict minimum. Les sécheurs que l'on utilise done aujourd'hui [4] sont constitués par des dispositif́s qui pulvérisent la caséine dans le courant d'air chaud et qui en même temps effectuent un véritable classement des particules, les plus grosses retombant dans l'appareil afin d'être pulvérisées plus finement, tandis que les plus petites sont entraînées par le courant d'air chaud et très rapidement séchées. On obtient dans ces conditions une poudre ne renfermant pas plus de 5 à $7 \%$ d'humidité, qui est recueillie dans des cyclones et pulvérisée une dernière fois à la finesse désirée.

En résumé, les avantages qui sont, d'après l'auteur du procédé, inhérents à cette nouvelle méthode sont les suivants :

10 Mélange immédiat et uniforme du lait et de l'acide ;

$2^{\circ}$ Séparation rapide et régulière de la caillebotte du sérum ;

$3^{\circ}$ Emploi d'acide chlorhydrique qui donne des sels facilement solubles avec les constituants inorganiques de la caséine ;

$4^{\circ}$ Lavage uniforme et efficace et élimination des impuretés solubles contenues dans la caséine ;

$5^{\circ}$ Pressage au moyen d'un appareil continu qui évite la formation de grands agrégats ;

$6^{\circ}$ Séchage rapide des petites particules de caséine qui évite la 
formation de peaux superficielles à l'abri desquelles l'hydrolyse se développe ;

$7^{\circ}$ Obtention d'une caséine de qualité supérieure.

\section{RÉFÉRENCES}

[1] W. H. SHEFFieLd, Brevet a mériea in 1.716 .799 du 11 juin 1929

[2] R. W. Змгтн. Ind. Eng. Chem., t. XXVI, 1934, p. 819.

[3] Maurice BEAu. La caséine, $1^{\text {re }}$ et $2^{\circ}$ partie. Le Lait, éditeur, 1932.

[4] F. L. ChappeLL. Brevet a méricain 1.892.233 du 27 déce mbre 1932.

\section{LES SAVEURS ET ODEURS ANORMALES DU LAIT}

$$
\text { par }
$$

Jean PIEN

Docteur ès-Sciences

Ingénieur chimiste (I.C.R.)

Directeur des Laboratoires des " Fermiers Réunis"

\author{
et \\ S. HERSCHDOEREER \\ Docteur ès-Sciences
}

(Vienne)

(Fin)

\section{L'HYDROLYSE DIASTASIQUE DE LA MATIËRE GRASSE DU LAIT}

Nous sommes toujours ici dans ce que nous avons appelé le cinquième groupe de causes des saveurs et odeurs anormales du lait, consistant en des modifications d'origine diastasique des éléments normalement contenus dans le lait. Nous avions divisé ces altérations en deux groupes : les oxydations qui mènent au goût d'huilage (que nous avons étudiées) et l'hydrolyse qui mène à la rancidité dont nous allons maintenant dire quelques mots.

Il est nettement établi depuis les travaux classiques de Porcher que la rancidité est bien un phénomène d'hydrolyse. Nous savons d'autre part que l'hydrolyse de la matière grasse du lait consiste en une décomposition des graisses en glycérine et acides gras parmi lesquels l'acide butyrique, cause du goût de rance et même d'une certaine saveur amère.

Nous savons encore que cette hydrolyse, qui peut être l'œuvre de certaines bactéries, est le plus souvent d'origine diastasique et nous connaissons la diastase responsable : la lipase.

a) Présence de la lipase dans le lait. - Le lait de vache normal paraît être assez pauvre en lipase. Il n'y a pas concordance de vues chez les divers auteurs à ce sujet. Pour Leroy et S. PaLmer [48] la lipase serait absente dans le lait de vache normal et n'existerait que dans les laits de lactation avancée à la suite d'une lipogénèse plus ou moins intense au niveau de la glande mammaire. DAviEs au contraire considère comme normale, dans le lait de vache, l'existence d'une lipase spécifique. 